\title{
Perioperative dynamic alterations in peripheral regulatory $T$ and $B$ cells in patients with hepatocellular carcinoma
}

Tianxiang Chen ${ }^{1,2}$, Dongli Song ${ }^{3}$, Zhihui Min ${ }^{3}$, Xiangdong Wang ${ }^{3}, \mathrm{Yu} \mathrm{Gu}^{4}$, Bajin Wei ${ }^{1,2}$, Jia Yao ${ }^{1,2}$, Kangjie Chen ${ }^{1,2}$, Zhijun Jiang ${ }^{1,2}$, Haiyang Xie ${ }^{1,2}$, Lin Zhou ${ }^{1,2}$ and Shusen Zheng ${ }^{1,2^{*}}$

\begin{abstract}
Background: Intratumoral and circulating regulatory T cells (Tregs) have been shown to be critical in the pathogenesis of hepatocellular carcinoma (HCC). However there is limited knowledge on the alterations of regulatory $B$ cells (Bregs). We here investigated perioperative dynamic alterations of peripheral circulating Tregs and Bregs in HCC patients to reveal the relationship between regulatory lymphocytes and its clinical implications.

Methods: 36 patients with HCC, 6 with chronic hepatitis B infection and 10 healthy donors were enrolled for this study. Frequencies of peripheral Tregs and Bregs were measured by flow cytometry with antibodies against CD4, CD25, CD127, CD19 and IL-10 before, and after radical surgery. Then, clinical informatics of HCC patients was achieved through Digital Evaluation Score System (DESS) for the assessment of disease severity. Finally, we analysed correlations between digitalized clinical features and kinetics of circulating regulatory lymphocytes.

Results: Level of circulating $\mathrm{CD}^{+} \mathrm{CD} 25^{+} \mathrm{CD} 127^{-}$Tregs in $\mathrm{HCC}$ patients was significantly lower than that in healthy donors and patients with chronic hepatitis B infection before surgery, but was increased after surgery. Preoperative level of $\mathrm{CD} 19^{+} \mathrm{IL}-10^{+}$Bregs in HCC patients was also significantly lower than the other groups. However it dramatically was elevated right after surgery and remained elevated compared to controls (about 7 days after surgery, $P=0.04$ ). Frequency of circulating Tregs was correlated with circulating leukocytes, ferritin, and clinical features suggesting tumor aggressiveness including portal vein thrombosis, hepatic vein involvement and advanced clinical stages. Frequency of circulating Bregs was associated with Hepatitis B e Antigen (HBeAg) and Hepatitis B virus (HBV) DNA copy number. In addition, DESS was significantly and positively correlated with other staging systems.
\end{abstract}

Conclusion: Frequencies of peripheral Tregs and Bregs in HCC patients increased after surgery. These results suggest that a postoperative combination of therapies against Tregs and Bregs may be beneficial for better outcome of HCC patients after resection.

Keywords: regulatory T cells, regulatory B cells, hepatocellular carcinoma, surgery, dynamic alteration, lymphocytes, clinical informatics

\section{Background}

Regulatory $\mathrm{T}$ cells (Tregs) are a subpopulation of $\mathrm{CD} 4^{+}$ and $\mathrm{CD} 8^{+} \mathrm{T}$ cells with immune suppressive function. In cancer patients especially patients with hepatocellular carcinoma (HCC), Tregs contribute to the dampening of

\footnotetext{
* Correspondence: shusenzheng@zju.edu.cn

'Division of Hepatobiliary and Pancreatic Surgery, Department of Surgery, First Affiliated Hospital, School of Medicine, Zhejiang University, Hangzhou, China

Full list of author information is available at the end of the article
}

the antitumor immune response [1,2]. Patients undergoing hepatic resection for $\mathrm{HCC}$ with prominent Treg infiltration showed increased recurrence and worse prognosis. Intratumoral Tregs have further been proposed to be an independent prognostic factor in HCC patients by several publications. In combination with cytotoxic $\mathrm{T}$ cells, Tregs can predict prognosis more effectively $[3,4]$. In addition, increased $\mathrm{CD} 4^{+} \mathrm{CD} 25^{+}$ Tregs in the tumor microenvironment of HCC were found to be correlated with tumor size and vascular
C Biomed Central

(c) 2012 Chen et al; licensee BioMed Central Ltd. This is an Open Access article distributed under the terms of the Creative Commons Attribution License (http://creativecommons.org/licenses/by/2.0), which permits unrestricted use, distribution, and reproduction in any medium, provided the original work is properly cited. 
invasion [3-5]. On the other hand, Ormandy and others first reported peripheral $\mathrm{CD} 44^{+} \mathrm{CD} 25^{+}$Tregs were increased in HCC patients [6]. However, contradict results were also described by others [7].

Recently, regulatory B cells (Bregs), a new family of regulatory cells, were found to control immune responses at both innate and adaptive levels $[8,9]$. Expansion of Bregs was demonstrated to inhibit harmful immune responses in chronic inflammation by deactivation of effector $\mathrm{T}$ cells and natural Killer $\mathrm{T}$ cells $[10,11]$. Furthermore, the suppressive immune function of Bregs appears to be in contact-dependent and independent manner [12]. These immune regulatory mechanisms comprise of protection from lethal inflammation, modulation of the development of autoimmune diseases [13-15], and inhibition of anti-cancer response in various tumor models [9,16-18]. However, few studies assess the role of Bregs in HCC development.

Although compelling evidence has suggested the important roles of both Tregs and Bregs in tumor development, few researches described both of them together in HCC patient samples. In the present study, we investigated perioperative alterations of both circulating Tregs and Bregs in patients with HCC and their relations to clinical phenotypes were examined. Clinical phenotypes, as clinical informatics, were achieved by a Digital Evaluation Score System (DESS) for assessing the severity of patients [19]. Frequencies of both circulating Tregs and Bregs elevated after surgery. These results suggest that a combined deletion of both Tregs and Bregs may be essential for better prognosis of patients with HCC after surgery. We also found significant correlations between digitalized clinical features and both peripheral regulatory lymphocytes. Integration of clinical informatics and experimental results is a useful method to conduct translational research.

\section{Methods}

\section{Patient Population}

Of 230 candidates from outpatients and inpatients, the current case-controlled study recruited 36 patients with primary liver cancer, 6 with chronic Hepatitis B infection (CHB) as chronic liver disease control, and 10 healthy donors as normal controls. None of patients with liver cancer received any invasive treatments like transcatheter arterial chemoembolisation or radiation frequency ablation before tumor resection. Among patients with liver cancer, 32 were pathologically diagnosed as hepatocellular carcinoma, while 4 as combination of hepatocellular and cholangiocarcinoma. Additionally, patients with chronic HBV infection enrolled were Hepatitis B Surface Antigen-positive for greater than 6 months according to its definition [20]. Based on clinicapathological features, patients were divided into different clinical stages by tumor node metastasis (TNM) staging system [21]. This project was approved by the Ethical Committee of First Affiliated Hospital of School of Medicine of Zhejiang University (NO.2011-108) and conducted in compliance with the Helsinki Declaration. All the participants were explained the investigative nature of the study and signed an informed consent before entry into study.

\section{Samples collection and preparation}

Peripheral blood samples were intravenously drew and collected. Samples were collected immediately after admission before any intervention, 1-2 days and about 7 (5-9) days after tumor resection. Samples of patients with chronic HBV infection were collected when patient were just admitted without therapeutic intervention. Peripheral blood mononuclear cells (PBMC) were isolated according to previously study $[22,23]$. In brief, whole blood samples were overlaid onto Ficoll separation media (Shanghai Danfan Network Science \& Technology, Shanghai, China) after 1:1 dilution with Hank's Balanced Salted Solution (Gibco, CA, USA). PBMC were centrifuged for $20 \mathrm{~min}$ at X1900 rpm, collected at the plasma interface and washed thrice after centrifugation at X1000 rpm for $10 \mathrm{~min}$. PBMC were frozen and stored in liquid nitrogen till flow cytometry analysis.

\section{Flow cytometry analysis}

Flow cytometry analysis was conducted by FACS Aria II flow cytometer (BD Bioscience, San Diego, CA, USA). For surface staining, suspensions of PBMC were stained on ice using predetermined optimal concentrations of each antibody for $30 \mathrm{~min}$, and fixed using fixation buffer (BD PharMingen, San Diego, CA). Tregs identified with $\mathrm{CD} 4^{+} \mathrm{CD} 25^{+} \mathrm{CD} 127^{-}$expression were stained with human regulatory $\mathrm{T}$ cell Cocktail (BD PharMingen) [24] and Bregs identified with $\mathrm{IL} 10^{+} \mathrm{CD} 19^{+}$expression were stained with human anti-CD19 PerCp-Cy5.5 and Human anti-IL10 PE (BD PharMingen) [25]. Intracellular IL-10 analysis was performed by flow cytometry, as described previously $[25,26]$. Briefly, cells were resuspended $\left(2 \times 10^{6}\right.$ cells $\left./ \mathrm{ml}\right)$ in medium and stimulated with CpG (Invivogen, San Diego, CA) and CD40L (1 $\mu \mathrm{g} / \mathrm{ml}$; R\&D Systems, Minneapolis, MN), Phorbol-12myristate-13-acetate (PMA,50 ng/ml; Sigma), ionomycin (1 $\mu \mathrm{g} / \mathrm{ml}$; Sigma), Brefeldin A (1X solution/ml; BD Bioscience) right before staining and flow cytometry analysis. After surface staining, for IL-10 detection, Fc receptors were blocked using FcR-Binding inhibitor (eBioscience, San Diego, CA). Cells were fixed, permeabilized using a Cytofix/Cytoperm kit (BD PharMingen), and stained with monoantibody against IL-10 according to the manufacturer's instructions. Results are expressed as the frequency of Tregs or Bregs. 
Digital Evaluation Score System for Liver Cancer Digital Evaluation Score System (DESS) for patients with HCC was designed and implicated, as described previously with slight modifications [19]. DESS was established by senior surgeons who are specialized in hepatobiliary and pancreas disease to switch clinical pearls and materials of HCC patients into digitalized information. Briefly, DESS was integrated with descriptive information of patient history, signs, physical examination, clinical imaging, and pathology and laboratory tests. Of them, 165 clinical variables selected from HCC patients were included in DESS and divided into different sections such as history (Additional file 1 Table S1), signs and physical examination (Additional file 2, Table S2), combined laboratory test (Additional file 3 Table S3), imaging (Additional file 4 Table S4) and pathology (Additional file 5 Table S5). Severity of each variable was scored and calculated as 0,1 , 2 and 4 (Additional file 1, 2, 3, 4). The maximal value of score 4 means far more above physiological range or much more critical condition, while the minimal value of score 0 indicates the variable is within physiological range. Several variables were 0 or 4 like "fatigue", "enlargement of lymph nodes" and "goblin", because they are either lack of standard discrimination criteria or subdivision relies too much on patient's or physician's personal judgment. The value of 3 was specially excluded, since exponential values could better amplify distance among different severity levels [19]. Variables of laboratory tests in DESS were scored on basis of the results of preoperative measurements after patient admission without clinical treatment. After clinical data was transformed into points of each variable and put them together, the total score of DESS ranged from 0 to 660 points, higher scores in our design indicate a severer condition.

\section{Data analysis}

All values were expressed as mean \pm SEM. Statistical analysis was applied by SPSS software (SPSS 18.0; SPSS Inc; Chicago, IL). Frequencies of peripheral Tregs and Bregs among groups were analysed with one way ANOVA, followed by an unpaired student's $t$-test. Ranked data as single variable scores of DESS was compared by Mann-Whitney test. Correlations between DESS scores and frequencies of Tregs and Bregs and between the frequency of circulating total lymphocytes and that of Tregs and Bregs were performed by Spearman's rho test and Pearson's test as appropriate. $P<$ 0.05 was considered as statistically significant.

\section{Results}

\section{Perioperative alterations of peripheral Tregs and Bregs}

Frequency of peripheral Tregs in HCC patients before surgery were significantly lower than that in the healthy $(P=0.002$; Figure $1 \mathrm{~B})$ and $\mathrm{CHB}$ patients $(P=0.02$;
Figure 1B). 1-2 days after surgery, frequency of Tregs was not different to the original level $(P=0$. 43). However, a significant elevation of frequency of Tregs was observed about 7 days after tumor resection, as compared with that before the operation $(1.38 \pm 0.12 \%$ in $\mathrm{HCCa} 7$ versus $1.14 \pm 0.09 \%$ in $\mathrm{HCCb}, P=0.04$; Figure 1). Frequency of Tregs of HCC patients about 7 days after surgery was similar to that of patients with $\mathrm{CHB}(P$ $=0.19)$ though still lower than the healthy $(P=0.047)$.

Frequencies of Bregs in the patients with $\mathrm{CHB}$ were significantly higher than those in the healthy and preoperative HCC patients $(P<0.05$; Figure 2$)$. Frequency of Bregs of HCC patients was significantly elevated by time after tumor resection $(1.88 \pm 0.23 \%$ in $\mathrm{HCCa} 1$ versus $3.48 \pm 0.53 \%$ in $\mathrm{HCCa} 7$, $P<0.001$; Figure 2), even significantly higher than that in $\mathrm{CHB}$ patients ( $\mathrm{HCCa} 7$ versus $\mathrm{CHB}, P=0.04$; Figure 2).

\section{Circulating kinetics of lymphocytes with Tregs and Bregs} Frequency and number of circulating lymphocytes of HCC patients considerably declined 1-2 days after

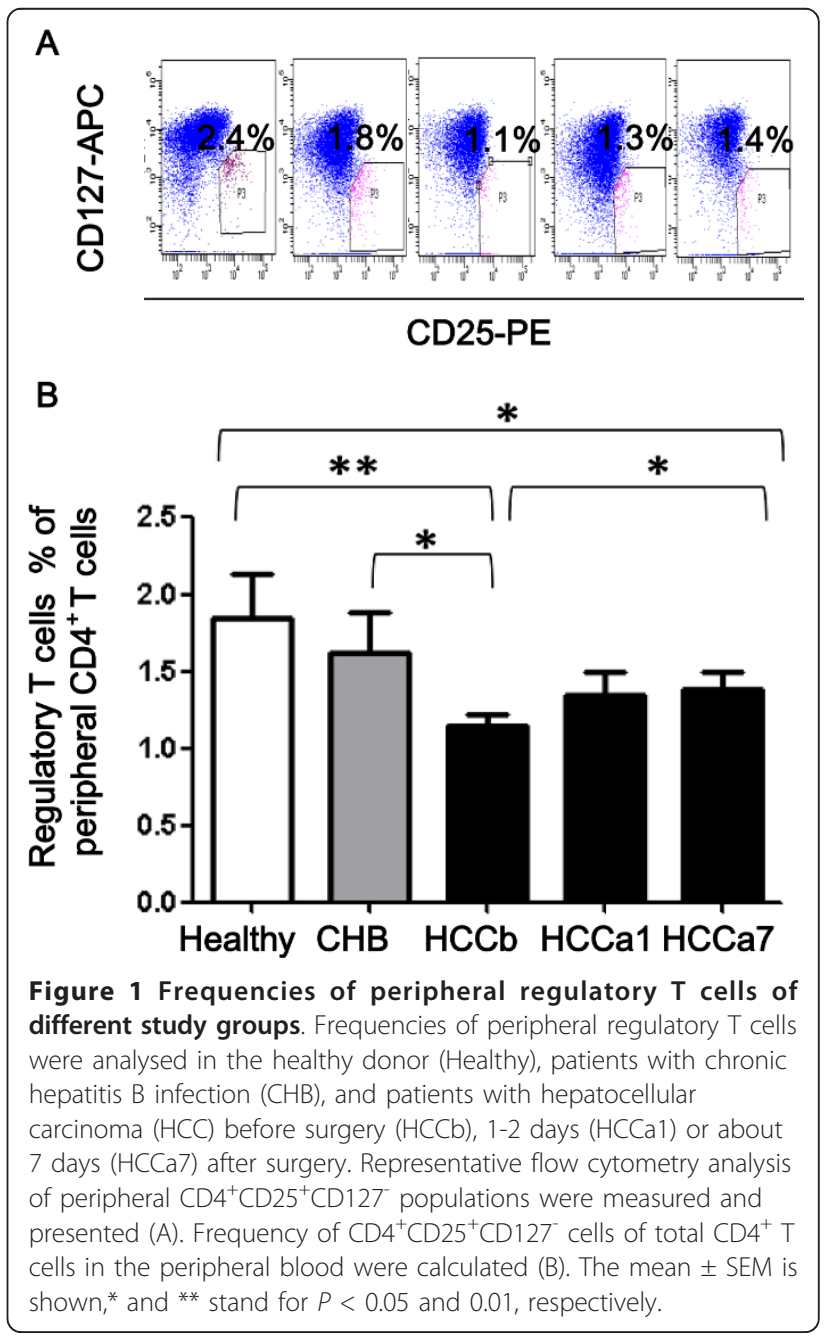




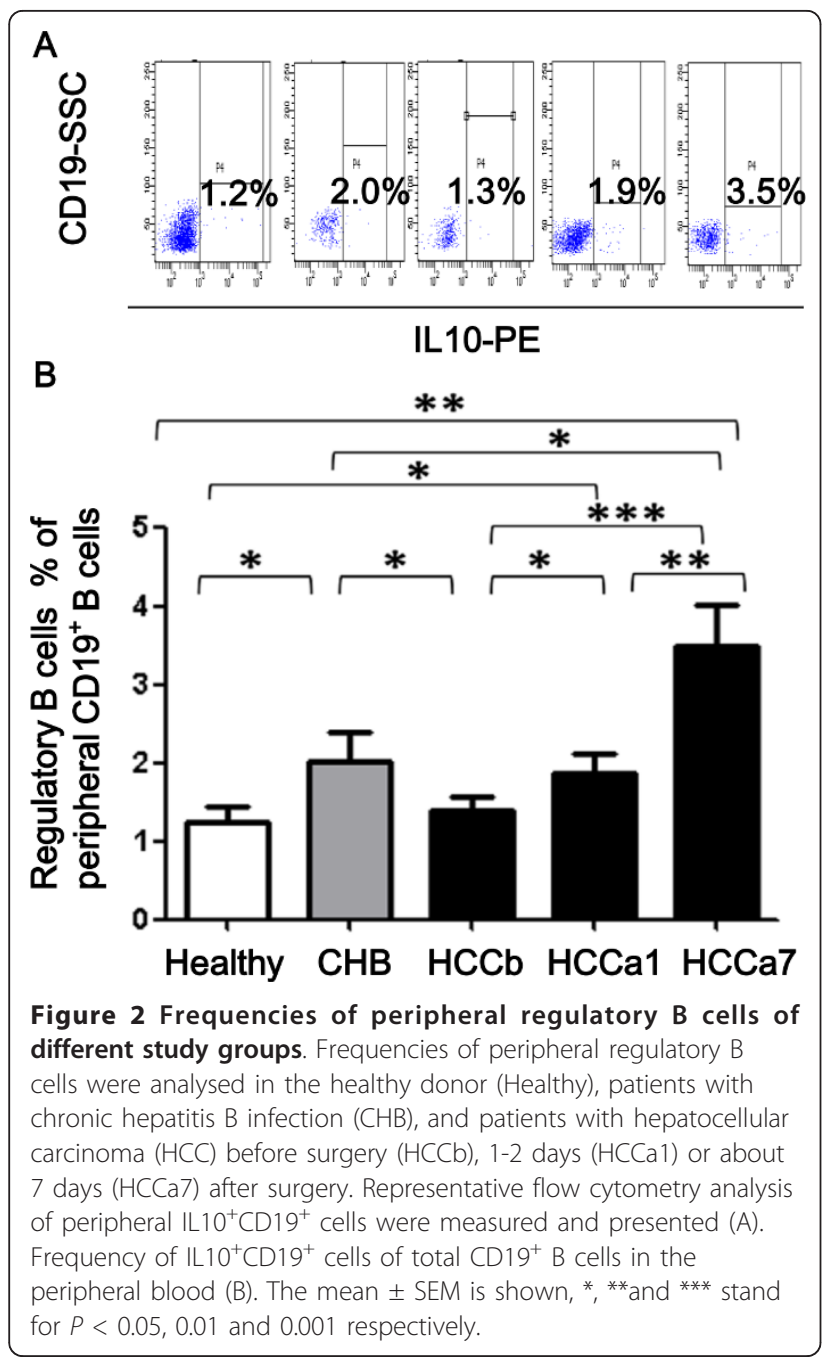

surgery, as compared with those before surgery $(P<$ 0.01 ; Figure 3$)$. However, it significantly elevated about 7 days after surgery, comparing with that 1-2 days after surgery $(P<0.001$; Figure 3$)$. The number of circulating lymphocytes about 7 days after surgery had no difference from the preoperative level. Frequency of circulating lymphocytes was correlated with the frequency of Tregs to some extent $(r=0.263, P=0.028)$.

\section{Acquisition of General features of study population through DESS}

All HCC patients $(n=36)$ were performed radical hepatic resection. Clinical informatics of $\mathrm{HCC}$ patients was digitalized through DESS as introduced in Methods. The mean value of HCC Patients' total score was $102.4 \pm 3.9$. Of them, $72 \%$ were stage I and the rest were more advanced. Various clinical features evaluated by DESS were compared between patients at mild stage (I) and advanced stage (II-IV). More than 10 features had higher DESS scores in patients at advanced stage $(P<0.05$ or 0.01 ; Table 1$)$. The mean rank

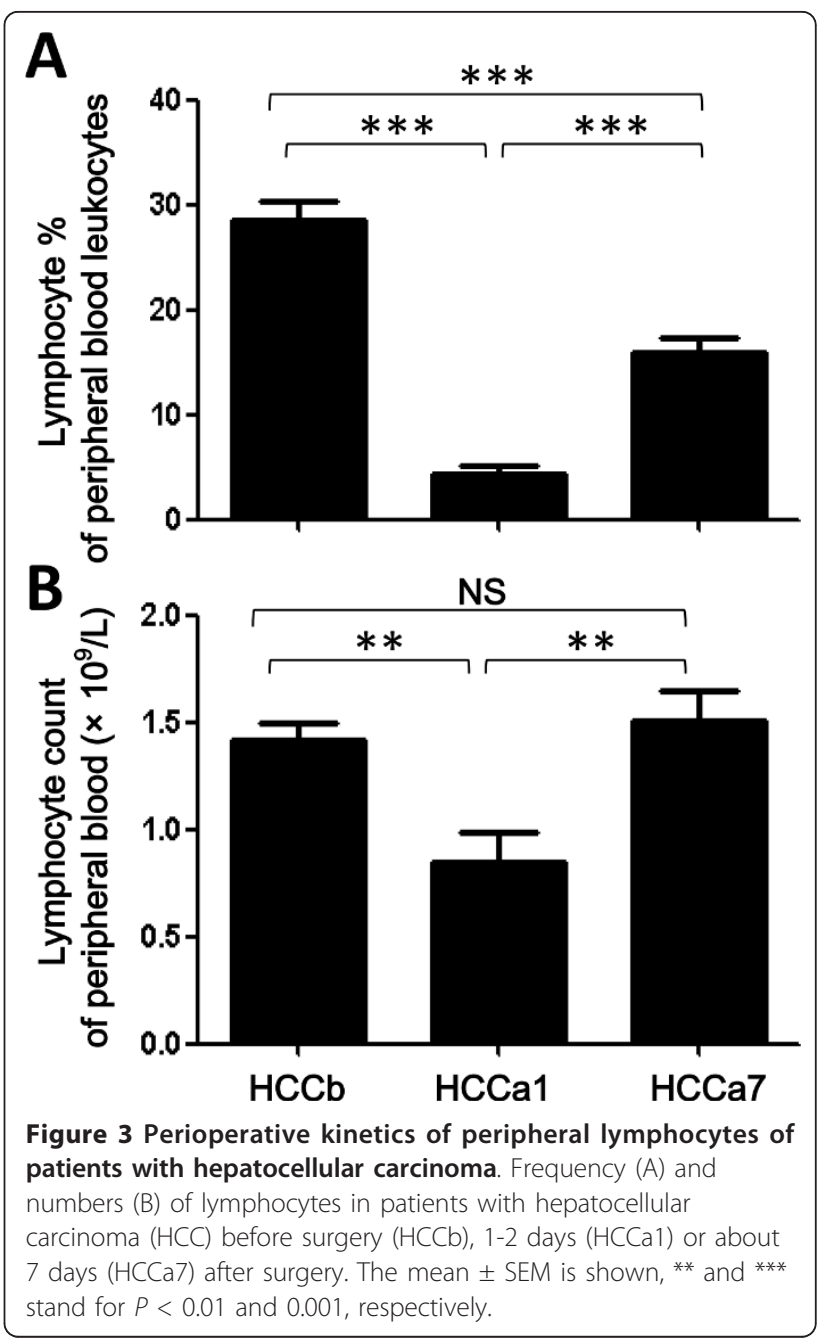

of total scores of patients at advanced stage was also significantly higher than that at mild stage (24.3 in advanced stage group versus 16.3 in mild stage group, $P=0.04$ ).

\section{Correlation between DESS scores and other clinical staging systems}

To better validate the value of DESS, patients were also staged using other 4 clinical staging systems: Liver Cancer Study Group of Japan/Tumor-Node-Metastasis Staging System(TNM/LCSGJ) [27], Child-Pugh Grading System [28], Barcelona Clinic Liver Cancer Staging System (BCLC) [29], and Cancer of Liver Italian Program Score (CLIP) [30]. The latter four systems are widely applied for the evaluation of liver cancer. Details of comparison between systems were summarized in Table 2. Significant and positive correlation between DESS and other staging systems was found $(P<0.05$ or 0.01 ; Table 2). There was a significant and strongest correlation between BCLC and DESS $(r=0.501, P=0.002)$, while not between Child-Pugh Grade and DESS. 
Table 1 Difference of clinical features between HCC patients at low and high stages of TNM (AJCC).

\begin{tabular}{cccc}
\hline Variables & \multicolumn{2}{c}{ Mean Rank } & $P$ \\
& $\begin{array}{c}\text { Stage } \\
\text { Low }\end{array}$ & $\begin{array}{c}\text { Stage } \\
\text { High }\end{array}$ & \\
\hline Platelet $\times 10^{9} / \mathrm{L}$ & 16.77 & 23.00 & 0.034 \\
Thrombocytocrit \% & 17.19 & 21.90 & 0.027 \\
Cystatin C $\mathrm{mg} / \mathrm{L}$ & 16.08 & 24.80 & 0.003 \\
Albumin/Globin & 16.38 & 24.00 & 0.032 \\
High density lipoprotein mmol/L & 15.88 & 25.30 & 0.001 \\
$\alpha$-fetoprotein ng/ml & 16.46 & 23.80 & 0.046 \\
Ferritin ng/ml & 15.62 & 26.00 & 0.004 \\
Numbers of up-regulated tumor & 15.48 & 26.35 & 0.003 \\
markers & & & \\
Multiple Satellite nodules & 16.77 & 23.00 & 0.053 \\
Tumor encapsulation & 16.88 & 22.70 & 0.022 \\
score of Pathology section & 15.58 & 26.10 & 0.007 \\
Score of Tumor markers & 16.35 & 24.10 & 0.042 \\
Total score & 16.27 & 24.30 & 0.040 \\
\hline
\end{tabular}

Stage low: TNM (AJCC) stage I. Stage high: TNM (AJCC) stage II-IV.

HCC: Hepatocellular carcinoma; TNM (AJCC): Tumor-Node-Metastasis Staging System (American Joint Committee on Cancer).

\section{Correlation between clinical features and circulating Tregs and Bregs}

Correlations of frequencies of peripheral Tregs and Bregs with DESS scores of clinical features were further examined in HCC patients (Table 3). High frequency of circulating Tregs was significantly correlated with the history of HBV infection $(P=0.031)$, circulating leukocytes $(P=0.029)$. The history of weight loss, plasma levels of ferritin, portal vein tumor thrombosis, hepatic vein invasion, and clinical stages evaluated by TMN systems or $\mathrm{BCLC} \operatorname{scores}(P<0.05$ or 0.01 ; Table 3$)$ were inversely associated with frequency of circulating Tregs. At the same time, frequency of circulating Bregs has a positive correlation with $\mathrm{HBeAg}(P=0.03)$ and $\mathrm{HBV}$ DNA copy number to some extent $(P=0.028)$.

Table 2 Correlations between total DESS scores and clinical stages of HCC patients.

\begin{tabular}{lcc}
\hline & \multicolumn{2}{c}{ Total DESS scores } \\
Staging system & $\boldsymbol{r}$ & $\boldsymbol{P}$ \\
\hline TNM (AJCC) stage & 0.365 & 0.029 \\
TNM (ICSGJ) stage & 0.364 & 0.029 \\
CLIP score & 0.474 & 0.004 \\
BCLC stage & 0.501 & 0.002 \\
Child-Pugh grade & -0.121 & 0.482
\end{tabular}

DESS: Digital evaluation scoring system; HCC: Hepatocellular carcinoma; TNM (AJCC): Tumor-Node-Metastasis Staging System (American Joint Committee on Cancer); TNM (LCSGJ): Tumor-Node-Metastasis Staging System (Liver Cancer Study Group of Japan); CLIP: Cancer of the Liver Italian Program Score; BCLC: Barcelona Clinic Liver Cancer Staging System.
Table 3 Correlations of clinical features of HCC patients with circulating Tregs and Bregs.

\begin{tabular}{cccccc}
\hline DESS evaluated & \multicolumn{2}{c}{ Tregs } & \multicolumn{2}{c}{ Bregs } \\
Clinicopathological Characteristic & $\boldsymbol{r}$ & $\boldsymbol{P}$ & $\boldsymbol{r}$ & $\boldsymbol{P}$ \\
\hline Weight Loss & -0.339 & $0.043^{*}$ & - & - \\
HBV Infection History & 0.360 & $0.031^{*}$ & - & - \\
Leucocyte & 0.363 & $0.029^{*}$ & - & - \\
Hepatitis B e Antigen & - & - & 0.527 & $0.030^{*}$ \\
HBV DNA Copy number & - & - & 0.532 & $0.028^{*}$ \\
Ferritin & -0.451 & $0.006^{* *}$ & - & - \\
Numbers of up-regulated tumor markers & -0.376 & $0.024^{*}$ & - & - \\
Portal vein tumor thrombosis & $-.408^{*}$ & $0.014^{*}$ & - & - \\
Hepatic vein invasion & $-.413^{*}$ & $0.012^{*}$ & - & - \\
BCLC score & -0.443 & $0.007^{* *}$ & - & - \\
TNM stage(AJCC) & -0.347 & $0.038^{*}$ & - & - \\
\hline
\end{tabular}

(*, ** stands for $P<0.05,0.01$, respectively)

\# Tested tumor markers: $\alpha$-fetoprotein; cancer antigen 125; cancer antigen 153; cancer antigen 199; Ferritin; Carcino-embryonic antigen; prostate specific antigen.

HCC: Hepatocellular carcinoma; Tregs: Regulatory T cells; Bregs: Regulatory B cells; HBV: Hepatitis B virus; BCLC: Barcelona Clinic Liver Cancer Staging System; TNM (AJCC): Tumor-Node-Metastasis Staging System (American Joint Committee on Cancer).

\section{Discussion}

Tregs, as a functionally unique subgroup of $\mathrm{T}$ lymphocytes, can suppress effective antitumor immune responses of various malignancies, including $\mathrm{HCC}$ $[31,32]$. The higher infiltration of Tregs in the local tumor microenvironment was found to correlate with poor prognosis of patients with HCC [3]. Likewise, increased frequency of peripheral Tregs was also shown to correlate with poor outcome [33]. However, unlike intratumoral Tregs, which were consistently reported to be increased in HCC patients, descriptions about frequency of peripheral Tregs were uncertain $[5,6,34]$. Since $\mathrm{CD} 4^{+} \mathrm{CD} 25^{+} \mathrm{CD} 127^{-}$was proved to be a classic surface marker for purified Tregs with highly suppressive function [35], Tregs were identified with CD4 ${ }^{+} \mathrm{CD} 25^{+} \mathrm{CD} 127^{-}$in the present study. Bregs are another special class of regulatory lymphocytes that modulating immune response. They have been suggested to be engaged in repressing autoimmune disease, chronic inflammatory diseases $[13,14]$ and promoting tumor progression $[16,18]$. However, most current studies about Bregs were conducted in animal models and little was known on disease specific patterns of peripheral Bregs in HCC patients. In this study, we investigated the dynamic frequencies of both peripheral Tregs and Bregs among HCC patients during the perioperative period.

Our data showed that the peripheral frequency of Tregs in HCC patients before surgery was significantly lower than control groups, but increased after tumor resection. No statistical difference were found between 
the healthy and CHB patients. Therefore, it indicates that comparatively lower levels of peripheral Tregs in HCC patients may be associated with HCC itself, rather than the chronic inflammatory phase. One hypothesis forwarded includes recruitment of peripheral Tregs to the local environment[36]. The migration of peripheral Tregs in HCC patients has been suggested to be related to the production of chemokines from HCC [37].

The present results demonstrated that the peripheral frequency of Bregs was higher in CHB patients as compared with healthy donors and HCC patients. It is possible that the systemic inflammatory state caused by hepatitis induces the expansion of peripheral Bregs. Similarly, the frequency of Bregs was dramatically increased in HCC patients after surgery, with the level higher than $\mathrm{CHB}$ patients 7 days following resection. To our knowledge, we are the first to show the perioperative dynamic changes to circulating Tregs and Bregs in HCC patients and found a similarly increased pattern of them after radical surgery. Further studies are needed to clarify such elevation is resulted from the removal of tumor or extensive liver surgery.

HCC may produce abundant disease specific cytokines and chemoattractants including IL-8 [38] and CCL 20 [37], some of which are responsible for the "homecoming" signals to orient regulatory lymphocytes into the tumor. After surgery, Tregs and Bregs may be temporally stocked in the peripheral circulation due to the descent of tumor chemoattractants. Bregs play an important role in the prevention of inflammation, autoimmune response $[39,40]$ and antitumor effects [17]. Hence, Bregs are probably involved in the local balance of immune tolerance and immune suppression in HCC like Tregs. To clarify the disease-specific dynamic profile of Tregs and Bregs in HCC, perioperative frequency of peripheral Tregs and Bregs in patients with pancreatic cancer was also measured. We found similar pattern but relatively altered degree of Tregs and Bregs in patients with pancreatic cancer (data unshown).

Accumulating evidences showed interaction between Tregs and Bregs in tumor microenvironment. For example, Bregs in the lung metastasis from breast cancer have been reported to induce conversion of resting CD4 ${ }^{+} \mathrm{T}$ cells to Tregs to support metastatic growth. These tumor-evoked Bregs express constructively activated stat3 and B7-H1 [16]. In additions, Bregs are involved in anti-inflammatory process through the promotion of Tregs in HCC, like those reported in autoimmune disease [41]. Our previous work discovered that the expression of B7-H1 and IL10 was up-regulated in HCC tissues [42]. Similar interactions between Breg and Tregs mentioned above may exist in human HCC development as well. Collectively, the postoperative increase of peripheral Tregs and Bregs in HCC patients might worsen the host immune system due to their established immune suppressive capabilities after surgery. This immunopathological condition of body caused by regulatory lymphocytes will promote tumor metastasis and recurrence. This could also partially explain the unsatisfactory outcome of HCC patients who even received radical surgery. Strategies against Tregs have been proved to enhance anti-cancer immunity [43]. Elimination of Bregs have also been suggested to be useful in the clearance of established tumor[44]. Therefore, a comprehensive adjuvant immunotherapy targeting both Tregs and Bregs may be beneficial for improving prognosis of post-surgery HCC patients.

Total number and percentage of circulating lymphocytes were initially deceased after surgery, but recovered within a week. During this period, a rapid proliferation of lymphocytes may occur. It was found that stem cells including mesenchymal stem cells could proliferate after surgery. Various growth factors produced by stem cells like heme oxygenase-1 could stimulate the expansion of Tregs and Bregs [45].

The application of clinical informatics made it easier to analyse the correlation between extensive clinical phenotypes and frequencies of peripheral Tregs and Bregs. Ferritin released by melanoma was reported to induce IL10 production of lymphocytes and suppress immune responses [46]. We found serum ferrtin levels were correlated with circulating Tregs in HCC patients supporting previous studies. In addition, the negative correlation of Tregs with up-regulation of a panel of routinely tested 8 tumor markers $(\alpha$-fetoprotein, cancer antigen 125 , cancer antigen 153, cancer antigen 199, ferritin, carcino-embryonic antigen, prostate specific antigen) has been discovered. Intratumoral Tregs were found to be positively correlated with vascular invasion [3]. In contrast, our data further demonstrated that the frequency of peripheral Tregs was correlated with portal vein thrombosis and hepatic vein invasion, supporting its connection with HCC aggressiveness. We found frequency of peripheral Bregs was positively correlated with HBeAg and HBV DNA copy numbers. These indicate that active HBV infection may stimulate the accumulation of circulating Bregs. To further explore the mechanism associated with the interactions between Tregs and Bregs, mediators from both regulatory cells should be profiled and validated. Network biomarkers depicting protein-protein interactions within both regulatory cells should be investigated by the integration of knowledge on protein annotations, interaction, and signalling pathway [47,48]. Dynamic network biomarkers should be further correlated with clinical informatics, thereby to select not only disease specific but also disease stage specific biomarker [49]. DESS as a useful tool of clinical informatics can be modified leading to extensive clinical application in future [19]. 


\section{Conclusions}

We report that frequencies of both peripheral Tregs and Bregs in HCC patients were decreased before surgery and significantly elevated after resection. These results suggest that a postoperative combined strategy targeting Tregs and Bregs could be beneficial for HCC patients to improve their prognosis. The correlations discovered between peripheral regulatory lymphocytes and clinical features through DESS set an example of clinical translational medicine.

\section{Additional material}

Additional file 1: Table S1. Variables and point values used in DESS for liver cancer patient (History).

Additional file 2: Table S2. Variables and point values used in DESS for liver cancer patient (Signs and physical exams).

Additional file 3: Table S3. Variables and point values used in DESS for liver cancer patient (Laboratory test).

Additional file 4: Table S4. Variables and point values used in DESS for liver cancer patient (Imaging).

Additional file 5: Table S5. Variables and point values used in DESS for liver cancer patient (Pathology).

\section{List of abbreviations}

Tregs: Regulatory T cells; Bregs: Regulatory B cells; HCC: Hepatocellular carcinoma; DESS: Digital evaluation scoring system; HBeAg: Hepatitis B e Antigen; HBV: Hepatitis B virus; CHB: Chronic hepatitis B virus infection; PBMC: Peripheral blood mononuclear cells; TNM (AJCC): Tumor-NodeMetastasis Staging System (American Joint Committee on Cancer); TNM (LCSGJ): Tumor-Node-Metastasis Staging System (Liver Cancer Study Group of Japan); BCLC: Barcelona Clinic Liver Cancer Staging System; CLIP: Cancer of the Liver Italian Program Score.

\section{Acknowledgements}

This study was supported by National Basic Research Program of China (973 Program) (No.2009CB522403), the Foundation for Innovative Research Groups of the National Natural Science Foundation of China (No.81121002) and Zhejiang Provincial Natural Science Foundation of China (No.Y2100498). We sincerely appreciate the selfless support from all the patients enrolled in this study.

\section{Author details}

'Division of Hepatobiliary and Pancreatic Surgery, Department of Surgery, First Affiliated Hospital, School of Medicine, Zhejiang University, Hangzhou, China. ${ }^{2}$ Key Laboratory of Combined Multi-organ Transplantation, Ministry of Public Health, Key Laboratory of Organ Transplantation, Zhejiang Province, Hangzhou, China. ${ }^{3}$ Biomedical Research Center, Zhongshan Hospital, Fudan University, Shanghai, China. ${ }^{4}$ Department of Surgical Oncology, First Affiliated Hospital, School of Medicine, Zhejiang University, Hangzhou, China.

\section{Authors' contributions \\ TC, DS, ZM, XW performed and participated in analysis of laboratory experiments data. TC, XW, LZ, HX, ZJ and SZ participated in the design of experiments. TC, YG, BW, JY, and KC acquired, preserved clinical samples and participated in clinical data analysis. LZ, HX, ZJ and SZ provided administrative support and funded experiments. TC, XW, YG, KC and SZ drafted the manuscript. All authors have contributed and approved the final manuscript.}

\section{Competing interests}

All authors declare that they have no competing interests.
Received: 1 November 2011 Accepted: 25 January 2012

Published: 25 January 2012

\section{References}

1. Nishikawa H, Sakaguchi S: Regulatory T cells in tumor immunity. Int J Cancer 2010, 127:759-767.

2. Curiel TJ: Regulatory T cells and treatment of cancer. Curr Opin Immunol 2008, 20:241-246.

3. Gao Q, Qiu SJ, Fan J, Zhou J, Wang XY, Xiao YS, Xu Y, Li YW, Tang ZY: Intratumoral balance of regulatory and cytotoxic $T$ cells is associated with prognosis of hepatocellular carcinoma after resection. J Clin Oncol 2007, 25:2586-2593.

4. Chen KJ, Zhou L, Xie HY, Ahmed TE, Feng XW, Zheng SS: Intratumoral regulatory $T$ cells alone or in combination with cytotoxic $T$ cells predict prognosis of hepatocellular carcinoma after resection. Med Oncol 2011.

5. Lee WC, Wu TJ, Chou HS, Yu MC, Hsu PY, Hsu HY, Wang CC: The impact of CD4(+)CD25(+) T cells in the tumor microenvironment of hepatocellular carcinoma. Surgery 2011

6. Ormandy LA, Hillemann T, Wedemeyer H, Manns MP, Greten TF, Korangy F: Increased populations of regulatory $T$ cells in peripheral blood of patients with hepatocellular carcinoma. Cancer Res 2005, 65:2457-2464.

7. Yang XH, Yamagiwa $S$, Ichida $T$, Matsuda $Y$, Sugahara $S$, Watanabe $H$, Sato $Y$, Abo T, Horwitz DA, Aoyagi Y: Increase of CD4+ CD25+ regulatory T-cells in the liver of patients with hepatocellular carcinoma. J Hepatol 2006, 45:254-262.

8. Mauri C, Blair PA: Regulatory B cells in autoimmunity: developments and controversies. Nat Rev Rheumatol 2010, 6:636-643.

9. Inoue S, Leitner WW, Golding B, Scott D: Inhibitory effects of B cells on antitumor immunity. Cancer Res 2006, 66:7741-7747.

10. Mauri C, Ehrenstein MR: The 'short' history of regulatory B cells. Trends Immunol 2008, 29:34-40.

11. Mizoguchi A, Bhan AK: A case for regulatory B cells. J Immunol 2006, 176:705-710.

12. Vitale G, Mion F, Pucillo C: Regulatory B cells: evidence, developmental origin and population diversity. Mol Immunol 2010, 48:1-8.

13. Matsushita T, Yanaba K, Bouaziz JD, Fujimoto M, Tedder TF: Regulatory B cells inhibit EAE initiation in mice while other B cells promote disease progression. J Clin Invest 2008, 118:3420-3430.

14. Watanabe R, Ishiura N, Nakashima H, Kuwano Y, Okochi H, Tamaki K, Sato S, Tedder TF, Fujimoto M: Regulatory B cells (B10 cells) have a suppressive role in murine lupus: CD19 and B10 cell deficiency exacerbates systemic autoimmunity. J Immunol 2010, 184:4801-4809.

15. Yanaba K, Yoshizaki A, Asano Y, Kadono T, Tedder TF, Sato S: IL-10producing regulatory $\mathrm{B} 10$ cells inhibit intestinal injury in a mouse model. Am J Pathol 2011, 178:735-743.

16. Olkhanud PB, Damdinsuren B, Bodogai M, Gress RE, Sen R, Wejksza K Malchinkhuu E, Wersto RP, Biragyn A: Tumor-evoked regulatory B cells promote breast cancer metastasis by converting resting CD4 T cells to T-regulatory cells. Cancer Res 2011, 71:3505-3515.

17. DiLillo DJ, Matsushita T, Tedder TF: B10 cells and regulatory B cells balance immune responses during inflammation, autoimmunity, and cancer. Ann N Y Acad Sci 2010, 1183:38-57.

18. Schioppa T, Moore R, Thompson RG, Rosser EC, Kulbe H, Nedospasov S, Mauri C, Coussens LM, Balkwill FR: B regulatory cells and the tumorpromoting actions of TNF-alpha during squamous carcinogenesis. Proc Natl Acad Sci USA 2011, 108:10662-10667.

19. Chen H, Song Z, Qian M, Bai C, Wang X: Selection of disease-specific biomarkers by integrating inflammatory mediators with clinical informatics in AECOPD patients: a preliminary study. J Cell Mol Med 2011.

20. Ganem D, Prince AM: Hepatitis B virus infection-natural history and clinical consequences. N Engl J Med 2004, 350:1118-1129.

21. Chun YH, Kim SU, Park JY, Kim DY, Han KH, Chon CY, Kim BK, Choi GH, Kim KS, Choi JS, Ahn SH: Prognostic value of the 7th edition of the AJCC staging system as a clinical staging system in patients with hepatocellular carcinoma. Eur J Cancer 2011.

22. Barre-Sinoussi F, Chermann JC, Rey F, Nugeyre MT, Chamaret S, Gruest J, Dauguet C, Axler-Blin C, Vezinet-Brun F, Rouzioux C, et al: Isolation of a Tlymphotropic retrovirus from a patient at risk for acquired immune deficiency syndrome (AIDS). Science 1983, 220:868-871.

23. Bull M, Lee D, Stucky J, Chiu YL, Rubin A, Horton H, McElrath MJ: Defining blood processing parameters for optimal detection of cryopreserved 
antigen-specific responses for HIV vaccine trials. J Immunol Methods 2007, 322:57-69.

24. Hein F, Massin F, Cravoisy-Popovic A, Barraud D, Levy B, Bollaert PE, Gibot S: The relationship between $C D 4+C D 25+C D 127$ - regulatory $T$ cells and inflammatory response and outcome during shock states. Crit Care 2010, 14:R19.

25. Knippenberg S, Peelen E, Smolders J, Thewissen M, Menheere P, Cohen Tervaert JW, Hupperts R, Damoiseaux J: Reduction in IL-10 producing B cells (Breg) in multiple sclerosis is accompanied by a reduced naive/ memory Breg ratio during a relapse but not in remission. $J$ Neuroimmunol 2011.

26. Iwata Y, Matsushita T, Horikawa M, Dilillo DJ, Yanaba K, Venturi GM, Szabolcs PM, Bernstein SH, Magro CM, Williams AD, et al: Characterization of a rare IL-10-competent B-cell subset in humans that parallels mouse regulatory B10 cells. Blood 2011, 117:530-541.

27. Kawaoka T, Aikata H, Takaki S, Hashimoto Y, Katamura Y, Hiramatsu A, Waki K, Takahashi S, Kamada K, Kitamoto M, et al: Transcatheter chemoembolization for unresectable hepatocellular carcinoma and comparison of five staging systems. Hepatol Res 2010, 40:1082-1091.

28. Yuen MF, Chan AO, Wong BC, Hui CK, Ooi GC, Tso WK, Yuan HJ, Wong DK, Lai CL: Transarterial chemoembolization for inoperable, early stage hepatocellular carcinoma in patients with Child-Pugh grade A and B: results of a comparative study in 96 Chinese patients. Am J Gastroenterol 2003, 98:1181-1185.

29. Santambrogio R, Opocher E, Costa M, Barabino M, Zuin M, Bertolini E, De Filippi F, Bruno S: Hepatic Resection for "BCLC Stage A" Hepatocellular Carcinoma. The Prognostic Role of Alpha-Fetoprotein. Ann Surg Oncol 2011.

30. Kudo $M$, Chung $H$, Osaki Y: Prognostic staging system for hepatocellular carcinoma (CLIP score): its value and limitations, and a proposal for a new staging system, the Japan Integrated Staging Score (JIS score). Gastroenterol 2003, 38:207-215.

31. Zabala M, Lasarte JJ, Perret C, Sola J, Berraondo P, Alfaro M, Larrea E, Prieto J, Kramer MG: Induction of immunosuppressive molecules and regulatory $T$ cells counteracts the antitumor effect of interleukin-12based gene therapy in a transgenic mouse model of liver cancer. J Hepatol 2007, 47:807-815.

32. Patel SA, Meyer JR, Greco SJ, Corcoran KE, Bryan M, Rameshwar P: Mesenchymal stem cells protect breast cancer cells through regulatory $T$ cells: role of mesenchymal stem cell-derived TGF-beta. J Immunol 2010, 184:5885-5894.

33. Xiong B, Feng G, Luo S, Liang H, Qiu L, Zheng C, Liu X, Zhou G: Changes of CD4(+) CD25 (+) regulatory $T$ cells in peripheral blood in patients with hepatocellular carcinoma before and after TACE. J Huazhong Univ Sci Technolog Med Sci 2008, 28:645-648.

34. Feng $X$, Li B, Ye H, Long D: Increased frequency of CD4+CD25(high)FoxP3 + regulatory $T$ cells in patients with hepatocellular carcinoma. Arch Immunol Ther Exp (Warsz) 2011, 59:309-314.

35. Liu W, Putnam AL, Xu-Yu Z, Szot GL, Lee MR, Zhu S, Gottlieb PA, Kapranov P, Gingeras TR, Fazekas de St, Groth B, et al: CD127 expression inversely correlates with FoxP3 and suppressive function of human CD4 + T reg cells. J Exp Med 2006, 203:1701-1711.

36. Zou W: Regulatory T cells, tumour immunity and immunotherapy. Nat Rev Immunol 2006, 6:295-307.

37. Chen KJ, Lin SZ, Zhou L, Xie HY, Zhou WH, Taki-Eldin A, Zheng SS: Selective Recruitment of Regulatory T Cell through CCR6-CCL20 in Hepatocellular Carcinoma Fosters Tumor Progression and Predicts Poor Prognosis. PloS One 2011, 6:e24671.

38. Wang $Y$, Wang $W$, Wang $L$, Wang $X$, Xia J: Regulatory mechanisms of interleukin 8 production induced by tumor necrosis factor-alpha in human hepatocellular carcinoma cells. J Cell Mol Med 2011.

39. Mizoguchi A, Mizoguchi E, Takedatsu H, Blumberg RS, Bhan AK: Chronic intestinal inflammatory condition generates IL-10-producing regulatory B cell subset characterized by CD1d upregulation. Immunity 2002, 16:219-230.

40. Matsushita T, Horikawa M, Iwata Y, Tedder TF: Regulatory B cells (B10 cells) and regulatory $T$ cells have independent roles in controlling experimental autoimmune encephalomyelitis initiation and late-phase immunopathogenesis. J Immunol 2010, 185:2240-2252.

41. Carter NA, Vasconcellos R, Rosser EC, Tulone C, Munoz-Suano A, Kamanaka M, Ehrenstein MR, Flavell RA, Mauri C: Mice lacking endogenous
IL-10-producing regulatory B cells develop exacerbated disease and present with an increased frequency of Th1/Th17 but a decrease in regulatory T cells. J Immunol 2011, 186:5569-5579.

42. Geng L, Deng J, Jiang G, Song P, Wang Z, Jiang Z, Zhang M, Zheng S: B7$\mathrm{H} 1$ up-regulated expression in human hepatocellular carcinoma tissue: correlation with tumor interleukin-10 levels. Hepatogastroenterology 2011, 58:960-964.

43. Zhang HH, Mei MH, Fei R, Liao WJ, Wang XY, Qin LL, Wang JH, Wei L, Chen HS: Regulatory T cell depletion enhances tumor specific CD8 T-cell responses, elicited by tumor antigen NY-ESO-1b in hepatocellular carcinoma patients, in vitro. Int J Oncol 2010, 36:841-848.

44. Bahlo DSaA: B Lymphocytes in Cancer Immunology. Experimental and Applied Immunotherapy 2011, Part 2:37-57.

45. Mougiakakos D, Jitschin R, Johansson CC, Okita R, Kiessling R, Le Blanc K: The impact of inflammatory licensing on heme oxygenase-1-mediated induction of regulatory T cells by human mesenchymal stem cells. Blood 2011, 117:4826-4835.

46. Gray $C P$, Franco AV, Arosio P, Hersey P: Immunosuppressive effects of melanoma-derived heavy-chain ferritin are dependent on stimulation of IL-10 production. Int I Cancer 2001, 92:843-850.

47. Baumgartner C, OsI M, Netzer M, Baumgartner D: Bioinformatic-driven search for metabolic biomarkers in disease. J Clin Bioinforma 2011, 1:2.

48. Wang $X$, Liotta L: Clinical bioinformatics: a new emerging science. J Clin Bioinforma 2011, 1:1.

49. Wang $X$ : Role of clinical bioinformatics in the development of networkbased Biomarkers. J Clin Bioinforma 2011, 1:28.

doi:10.1186/1479-5876-10-14

Cite this article as: Chen et al:: Perioperative dynamic alterations in peripheral regulatory $\mathrm{T}$ and $\mathrm{B}$ cells in patients with hepatocellular carcinoma. Journal of Translational Medicine 2012 10:14.

\section{Submit your next manuscript to BioMed Central and take full advantage of:}

- Convenient online submission

- Thorough peer review

- No space constraints or color figure charges

- Immediate publication on acceptance

- Inclusion in PubMed, CAS, Scopus and Google Scholar

- Research which is freely available for redistribution

Submit your manuscript at www.biomedcentral.com/submit
C Biomed Central 